\title{
High-speed streams in the solar wind during the last solar minimum
}

\author{
Georgeta Maris ${ }^{1}$, Ovidiu Maris ${ }^{2}$, Constantin Oprea ${ }^{1}$, \\ Marilena Mierla ${ }^{1,3,4}$ \\ ${ }^{1}$ Institute of Geodynamics of the Romanian Academy, \\ RO-020032, Bucharest, Romania \\ email: gmaris@geodin.ro \\ ${ }^{2}$ Institute for Space Sciences, RO-077125 Bucharest, Romania \\ ${ }^{3}$ Royal Observatory of Belgium, Brussels, Belgium \\ ${ }^{4}$ Research Center for Atomic Physics and Astrophysics, \\ Faculty of Physics, University of Bucharest, Romania
}

\begin{abstract}
The paper presents a statistical analysis of the fast solar wind streams during the last prolonged minimum. Defining a minimum phase as the period with the monthly relative sunspot numbers (smoothed values) having a value of less than 20 , we considered for this analysis the interval February 2006 September 2010. The High-Speed Streams (HSSs) in the solar wind were determined by their main parameters: duration, maximum velocity, velocity gradient. A comparative analysis of the HSS dynamics during the last solar minimum with the previous solar minimum (1996-1997) concludes the paper.
\end{abstract}

Keywords. Sun: activity, (Sun:) solar wind

\section{Introduction}

Solar wind plays an important role in the heliospheric structure and dynamics and it is "the medium" through which all the solar perturbations are propagating towards the Earth. Lots of space missions were and still are dedicated to recording its composition, velocity, density and temperature, inside and, especially, outside of the terrestrial magnetosphere (WIND, ACE, Ulysses and SOHO). Solar wind plasma consists of electrons, protons, helium and heavier nuclei, which are carrying alongside them the solar magnetic field resulting in an interplanetary magnetic field. The average velocity of the solar wind plasma is $350 \mathrm{~km} / \mathrm{s}$ with a minimum of about $200 \mathrm{~km} / \mathrm{s}$ and a maximum over $1000 \mathrm{~km} / \mathrm{s}$. The high speed streams (HSSs) in the solar wind were measured by many recording earth-orbiting or solar-orbiting spacecraft. The fast solar wind $(>650 \mathrm{~km} / \mathrm{s})$ is characterized by a high temperature, a low density and a low mass flux, while the slow solar wind $(<400 \mathrm{~km} / \mathrm{s})$ is cooler, denser and has a larger mass flux. There are also other differences in composition, in protons and electrons temperature anisotropy, etc. between slow and fast solar wind (Schwenn 2006).

In this paper we present a statistical analysis of the HSSs during the last prolonged solar minimum in comparison with their statistics during the previous minimum.

\section{Data and Methods of Analysis}

\subsection{HSSs and their Parameters}

A lot of HSSs definitions were given by different authors and HSSs catalogues covering solar cycles nos. 20 - 23 were set up (Lindblad \& Lundstedt 1981; 1983; Lindblad et al. 
1989; Mavromichalaki et al. 1988; Mavromichalaki \& Vassilaki 1998; Maris \& Maris 2009; 2012). We used the same definition and selection procedure of the streams as Lindblad and Lundsted because it allows for a more precise determination of the HSS beginning and end: a 'high-speed stream' is a solar wind flow having $\Delta V 1>100 \mathrm{~km} / \mathrm{s}$ that lasted for two days, where $\Delta V 1$ is the difference between the smallest 3-hr mean plasma velocity for a given day $(V 0)$ and the largest 3-hr mean plasma velocity for the following day $(V 1)$.

From among the HSS parameters we used their maximum velocity $V_{\max }($ in $\mathrm{km} / \mathrm{s}$ ), the duration $d$, in days, and the maximum gradient of the velocity:

$$
\Delta V_{\max }=V_{\max }-V_{0}
$$

where $V_{0}$ - the minimum (pre-stream) solar wind velocity (in $\mathrm{km} / \mathrm{s}$ ).

\subsection{Minimum Phase of the 11-yr Solar Cycle}

The 11-yr solar cycle (SC), generally defined through the sunspot relative number, is the most evident periodicity that can be observed in all the solar atmospheric phenomena. The period of one solar cycle is defined as the interval between two successive minima of the Wolf curve. The length of approximately 11 years was statistically determined. It certainly represents the clearest periodicity observed also in all eruptive solar phenomena (flares, CMEs, solar active prominences etc.) that follow the 11-yr cycle evolution with their power and frequency of occurrence. It is best seen in the frequency indices' variability of some active solar phenomena.

We emphasize that the phases of the 11-yr solar cycle (minimum, ascendant, maximum and descendant phases) are not "rigid" components; they could have different durations and intensities in different cycles. Furthermore, maximum and minimum phases of the 11-yr cycle are referring to some intervals of time during which solar activity has a maximum or minimum level, respectively, unlike the momentary maximum or minimum of the 11-yr SC, that are referring to the maximum or minimum monthly (or annual) values, respectively, reached by some solar index during the cycle. We emphasize the main characteristics of the 11-yr solar cycle phases:

- The ascendant phase - the only interval of any cycle during which the influences of the solar cycles adjacent to it (the precedent and the following ones) are not present;

- The maximum phase - a phase with significant activity of the majority of solar phenomena having superimposed on it, in its second part, the beginning of the polar solar magnetic field reversal;

- The descendant phase - the most "complex" phase, when in the solar atmosphere and in the tachocline (underneath the convective zone) there are two magnetically opposite dipoles;

- The minimum phase - the apparent "quiet" phase when the old cycle (the old dipole) is using up its reserves (it's dying out) but in the sub-surface levels the new cycle is beginning as new active regions with the opposite polarity distribution.

We analyzed the HSS activity level during different phases of the 11-yr solar cycle in some previous papers (Maris \& Maris 2005; 2010; 2011). We have chosen the phase limits considering the maximum and minimum structure of the curve for the smoothed sunspot relative number, $\mathrm{W}$, in each $\mathrm{SC}$, the sunspot relative number being the standard parameter of the 11-year SC dynamics. We have considered the minimum phases as the intervals with the sunspot relative number $W<20$. According to this definition of the 11-year cycle minimum, we obtained for SCs 20-23 the phases specified in Table 1 .

The lengths of the minimum phases are more or less equal for SCs $20-23$, but the length of SC 24 minimum is more than double of the previous cycle minimum (that 
Table 1. Minimum phase durations of the solar cycles nos. 20 - 24 (calendar months) and the HSS numbers.

\begin{tabular}{|c|c|c|c|c|}
\hline Sc no. & $\begin{array}{c}\text { Minimum } \\
\text { Phase }(\mathrm{mm} \cdot \mathrm{yyyy})\end{array}$ & \begin{tabular}{|l} 
Duration \\
(months)
\end{tabular} & $\mid \begin{array}{c}\text { HSS } \\
\text { number }\end{array}$ & $\begin{array}{c}\text { HSS } \\
\text { no./month }\end{array}$ \\
\hline 20 & $01.1964-10.1965$ & 22 & 34 & 1.5 \\
\hline 21 & $04.1975-02.1977$ & 23 & 66 & 2.9 \\
\hline 22 & $02.1985-02.1987$ & 25 & 69 & 2.8 \\
\hline 23 & $05.1995-05.1997$ & 25 & 79 & 3.2 \\
\hline 24 & $02.2006-09.2010$ & 57 & 283 & 5.0 \\
\hline
\end{tabular}

being SC 23). The number of the HSSs recorded during the minimum phases (column 4 in Table 1), as well as the number of HSSs recorded per month (column 5 in Table 1) are not significantly different for SCs nos. $21-23$. The small values recorded in the minimum of cycle no. 20 (34, and 1.5, respectively) can be, at least partially, explained by the limited ways of recording the solar wind plasma outside the magnetosphere during that era. In complete contrast, after 1996, the ACE and SOHO space missions, along with WIND and Ulysses, have ensured for a continuous monitoring of the solar wind plasma in regions outside the terrestrial magnetosphere.

\section{HSS Statistics during SC 23 and SC 24 Minima}

Figs. 1 - 3 present the statistical distributions of the HSSs in intervals of their duration, maximum velocity and maximum velocity gradient, respectively for SCs 23 (left panels) and 24 (right panels) minima.
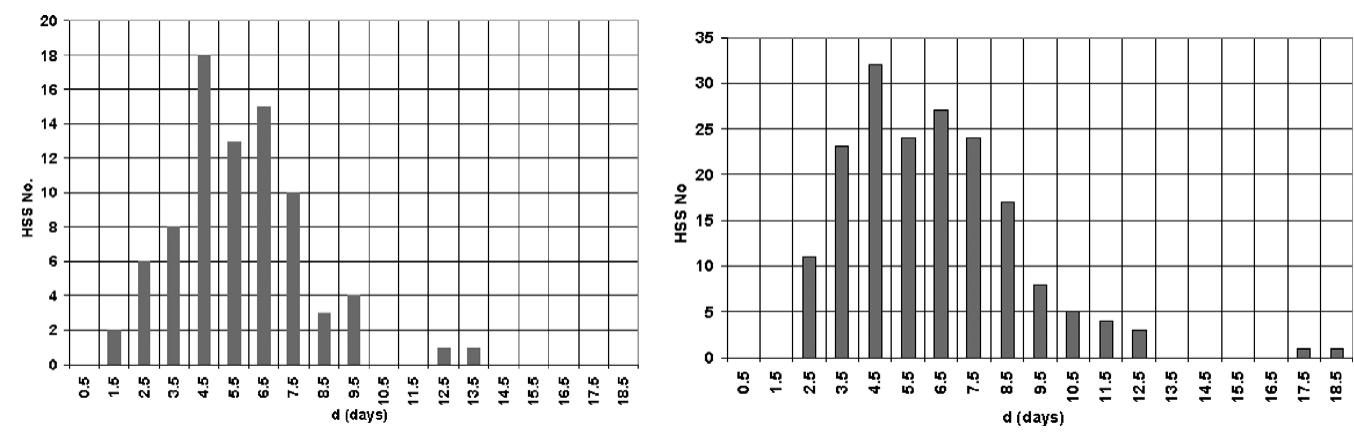

Figure 1. HSSs distributions by their durations during the minima of SC 23 (left panel) and SC 24 (right panel).

In Fig. 1 it is clearly seen that during SC 23 minimum, the majority of HSSs had durations of 4 - 8 days with peaks at 4.5 and 6.5 days, and during SC 24 minimum, the majority of HSSs had larger durations, of 3 - 9 days, with the same peaks at 4.5 and 6.5 days.

In Fig. 2 it is clearly seen that during SC 23 minimum, the maximum number of HSSs had $500 \leqslant V_{\text {max }} \leqslant 600 \mathrm{~km} / \mathrm{s}$ whereas during SC 24 minimum, the maximum number of HSS moved to the higher values of maximum velocity, $600 \leqslant V_{\max } \leqslant 700 \mathrm{~km} / \mathrm{s}$. 
We can remark in Fig. 3 that the maximum number of HSSs had $100 \leqslant \Delta V_{\max } \leqslant$ $300 \mathrm{~km} / \mathrm{s}$ during the SC 23 minimum, whereas the maximum number of HSSs is rather equally distributed in the three division of $100 \mathrm{~km} / \mathrm{s}$ of the $100-400 \mathrm{~km} / \mathrm{s}$ interval during the SC 24 minimum.
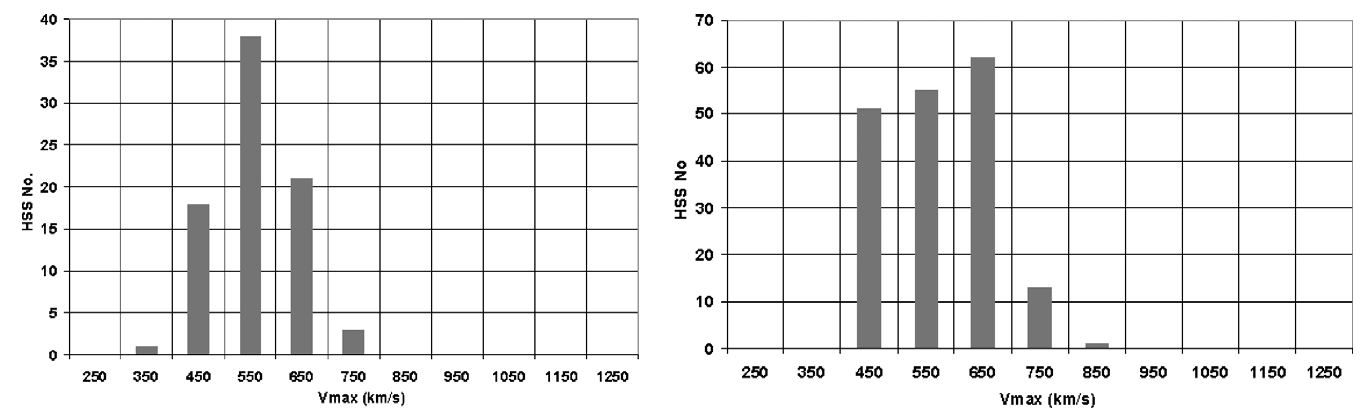

Figure 2. HSSs distributions by their maximum velocity during the minima of SC 23 (left panel) and SC 24 (right panel).
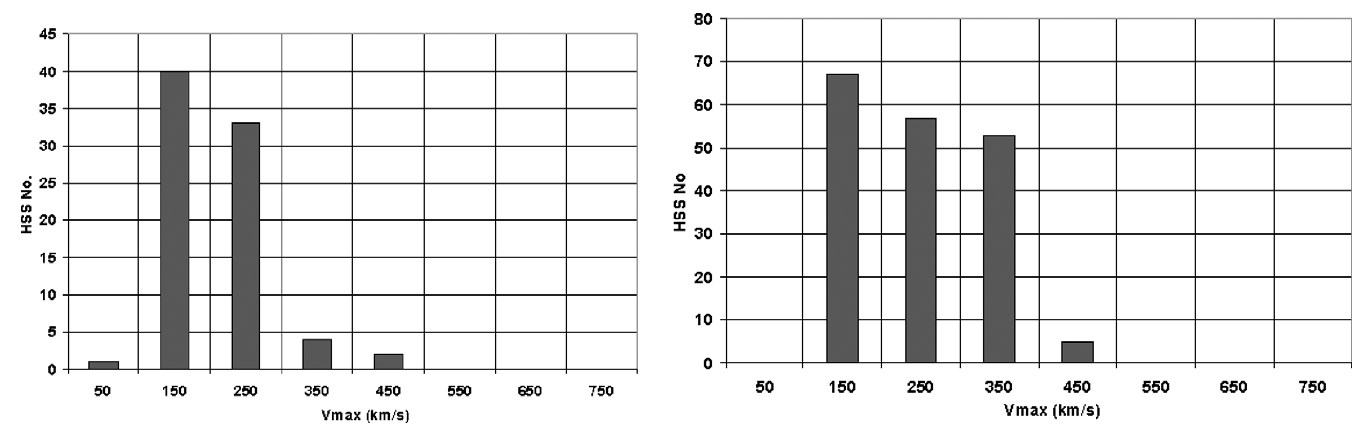

Figure 3. HSSs distributions by their maximum gradient velocity during the minima of SC 23 (left panel) and SC 24 (right panel).

A similar analysis for the minimum phases of all SCs 20 - 24 did not show any significant differences between odd and even SCs.

\section{Summary}

- The durations of the minimum phases for SCs 20-23 are quite equal (they lasted for 22 - 25 months), but the last minimum phase exceeded two times the previous minimum phases (57 months);

- The HSS number per minimum is slowly growing from one cycle to the next one, but it remains about 3/month for SCs 21 - 23. Frequencies of $1.5 \mathrm{HSSs} /$ months and about $5 \mathrm{HSSs} /$ months are opposite exceptions for the minimum phases of SC 20 and SC 24, respectively;

- Minimum phases situated after SCs with lower $W_{\max }$ (nos. 20 and 23) are more active in HSS occurrence.

Consequently, the minimum phases of the 11-yr solar cycles are not quiet intervals but they are periods with significant activity in the solar corona where especially more equatorial coronal holes could appear and could be sources of more energetic HSSs. 


\section{Acknowledgments}

The work of CO and MM was supported from the project TE no. 73/11.08.2010.

\section{References}

Lindblad, B. A. \& Lundstedt, H. 1981, Sol. Phys., 74, 197-206

Lindblad, B. A. \& Lundstedt, H. 1983, Sol. Phys., 88, 377-382

Lindblad, B. A., Lundstedt, H., \& Larsson B. 1989, Sol. Phys., 120, 145-152

Maris, O. \& Maris, G. 2005, Adv. Space Res., 35, 2129-2140

Maris, O. \& Maris, G. 2009, at: http://www.spaceweather.eu/, in Chap. Data Acces, or at: http://spacescience.ro/new1/HSS_Catalogue.html

Maris, G. \& Maris, O. 2010, Proc. IAU Symposium 264 Solar and Stellar Variability Impact on Earth and Planets, A.G. Kosovichev, A.H. Andrei and J.-P. Rozelot (Eds.), Cambridge Univ. Press., 359-362, doi: 10.1017/S1743921309992924

Maris, G. \& Maris, O. 2011, 3rd School and Workshop on Space Plasma Physics, I. Zhelyazkov, T. Mishonov (Eds.), AIP Conf. Proc. 1356, 177-191, doi: 10.1063/1.3598104

Maris, G. \& Maris, O. 2012, Chapter 7 in: Advances in Solar Terrestrial Physics, G. Maris \& C. Demetrescu (Eds.), Research Signpost Publ., Trivandrum, Kerala, India, ISBN: 978-81308-0483-5, 97-134, in press

Mavromichalaki, H., Vassilaki, A., \& Marmatsouri, E. 1988, Sol. Phys. 115, 345-365

Mavromichalaki, H. \& Vassilaki, A. 1998, Sol. Phys. 183, 181-200

Schwenn, R. 2006, Living Rev. Solar Phys. 3, http://www.livingreviews.org/lrsp-2006-2. 